\title{
Continuous output feedback stabilization for nonlinear systems based on sampled and delayed output measurements
}

\author{
Yanjun Shen ${ }^{1 *}$, Daoyuan Zhang ${ }^{1}$, Xiaohua $\mathrm{Xia}^{2}$ \\ 1 College of Electrical Engineering \& New Energy, China Three Gorges University, Yichang, Hubei, 443002, China \\ 2 Department of Electrical, Electronic and Computer Engineering, University of Pretoria, Pretoria 0002, South Africa
}

\begin{abstract}
Summary
This paper addresses the problem of output feedback stabilization for nonlinear systems with sampled and delayed output measurements. Firstly, sufficient conditions are proposed to ensure that a class of hybrid systems are globally exponentially stable. Then, based on the sufficient conditions and a dedicated construction continuous observer, an output feedback control law is presented to globally exponentially stabilize the nonlinear systems. The output feedback stabilizer is continuous and hybrid, and can be derived without discretization. The maximum allowable sampling period and the maximum delay are also given. At last, a numerical example is provided to illustrate the design methods.
\end{abstract}

Keywords: nonlinear systems; output feedback stabilization; hybrid systems; sampled-data; delayed measurements

\section{Introduction}

Recently, great progress has been made in the problem of design global asymptotic output feedback control laws for nonlinear systems. For example, the problem of global asymptotic stabilization by output feedback has been studied for a class of nonlinear systems [1]. The nonlinear terms considered in [1] admit an incremental rate depending on the measured output. In [2], the author introduced a technique to stabilize a fully linearizable nonlinear system. The technique was utilized in [3] and [4]. In [5], a linear output feedback controller with dynamic high gain was presented to globally regulate a class of nonlinear systems.

It should be noted that the above results concerned with output feedback stabilization are based on continuous time analysis. However, for a networked control system, the output is usually transmitted through a shared band-limited digital communication network. It is only available at discrete-time instants. Therefore, it is interesting to study output feedback stabilization for continuous systems with sampled and delayed measurements. More recently, three main approaches are proposed to deal with these problems. The first one is based on discrete time analysis by introducing a consistent approximation of the exact discretized model [6,7]. A quadratic observer coupled with a quadratic dynamic feedback was proposed to achieve quadratic approximated feedback linearization with stability [7]. The second one is based on continuous time analysis followed by discretization $[8,9,10,11]$. For example, sampled-data output feedback stabilization of nonlinear systems by using high-gain observers has been considered in [8, 9, 10]. In [11], multirate sampleddata output feedback control of a class of nonlinear systems was presented based on high gain

*Correspondence to: shenyj@ctgu.edu.cn 
observers. By carefully choosing the sampling period, the authors proposed a sampled-data output feedback controller to make the closed-loop systems regionally or globally stable [12]. The results obtained in [12] are based on the assumption that full-state are measurable. The third one is based on a mixed continuous and discrete time analysis without discretization [13, 14, 15, 16, 17, 18]. For example, based on the existence of a controller and a special Lyapunov function satisfying certain $\mathcal{L}_{2}$ gain conditions, the authors presented a sampled-data output feedback stabilizer to ensure that the closed-loop system is globally stable by using a hybrid system method [13]. It should be noted that the closed-loop system is required to transformed into the hybrid system introduced in [20]. In $[17,19]$, a state feedback law has been constructed to achieve global asymptotic stabilization for nonlinear systems under sampled and delayed measurements, and with inputs subject to delay and zero-order hold. There are few results on sampled-data output feedback stabilization for nonlinear systems in lower-triangular form with delayed output measurements, which motivates the present study.

In this paper, our aim is to design an output feedback stabilizer for a class of nonlinear systems with sampled and delayed measurements. Firstly, sufficient conditions are given to ensure that a class of hybrid systems are globally exponentially stable. The sufficient conditions are derived by constructing an iteration with a parameter. Then, based on the sufficient conditions and a dedicated construction continuous observer, an output feedback control law is presented to globally exponentially stabilize the nonlinear systems. The output feedback stabilizer is continuous and hybrid. It has simple and explicit form and can be derived without discretization. The maximum allowable sampling period and the maximum delay are also given.

This paper is organized as follows. In Section 2, some definitions are presented for hybrid systems. Then, global exponential stable sufficient conditions are given for the hybrid systems. In Section 3, continuous output feedback stabilizer are presented for a class of single-output nonlinear systems with sampled and time delayed measurements. Section 4 provides an example to illustrate the validity of the proposed design methods. Finally, the paper is concluded in Section 5.

\section{Global exponential stability for hybrid systems}

Let $\mathbb{R}^{n}$ denote $n$-dimension real space and $\mathbb{R}^{+}$denote 1 -dimension positive real space. For any $x \in \mathbb{R}^{n}$, let $\|x\|=\left(x^{T} x\right)^{1 / 2}$. For a continuous function $f: \mathbb{R} \rightarrow \mathbb{R}$ and $t \in \mathbb{R}$, let $\lim _{s \rightarrow t^{-}} f(s)=$ $\lim _{s \rightarrow t, s<t} f(s)$. For a matrix $P \in \mathbb{R}^{n \times n}, \lambda_{\max }(P)$ and $\lambda_{\min }(P)$ denote the largest and the smallest eigenvalues of $P$, respectively.

Consider the following system:

$$
\left\{\begin{array}{c}
\dot{x}(t)=f\left(x(t), x\left(t_{k}\right)\right), \\
t_{k+1}=t_{k}+T_{k+1}, \\
x\left(t_{k+1}+\tau_{k+1}\right)=\lim _{t \rightarrow t_{k+1}+\tau_{k+1}-x(t),}, \\
t \in\left[t_{k}+\tau_{k}, t_{k+1}+\tau_{k+1}\right), k \geq 0,
\end{array}\right.
$$

where $\left\{t_{k}\right\}_{k \in N}$ is a sequence of positive numbers defined by $t_{k+1}=t_{k}+T_{k+1}, T_{k+1}$ denotes the $k+1$ th sampling period, $\tau_{k}$ denotes time delay and has an upper bound $\tau$, that is $\tau_{k} \leq \tau$. Let $T_{\max }=\max \left\{T_{k}\right\}$ and $T_{\min }=\min \left\{T_{k}\right\}$. We also assume that $\tau \leq T_{\min }$, which can be taken non-strict with the understanding that in case the update instant $t_{k}+\tau_{k}$ coincides with the next transmission instant $t_{k+1}$, the update is performed before the next sample is taken. The function $f: \mathbb{R}^{n} \times \mathbb{R}^{n} \rightarrow \mathbb{R}^{n}$ is continuous. It should be noted that $x(t)$ is continuous on $t_{k+1}+\tau_{k+1}$, therefore, it is continuous on $\left[t_{0}, \infty\right)$.

\section{Remark 1}

Sampling arises simultaneously with input and output delays in many control problems, especially in control over networks. Similar systems to the form (1) arise frequently in certain applications in mathematical control theory and numerical analysis, which have been investigated in $[15,17,19$, $21,22,23,24]$. For example, the authors proposed a system-theoretic framework to study hybrid uncertain systems [21]. They also presented characterizations of robust global asymptotic output stability. In [15], observer was designed for certain classes of nonlinear systems with both sampled 
and delayed measurements by using a small gain approach. Continuous-discrete observers have also been studied for multi-input and multi-output state affine systems with sampled and delayed output measurements [24].

We next give the following definitions for the hybrid system (1).

\section{Definition 1}

Consider the system (1), for each $\left(t_{0}, x_{0}\right) \in \mathbb{R}^{+} \times \mathbb{R}^{n}$, there exists a piecewise continuous function $t \rightarrow x\left(t, t_{0}, x_{0}\right)$ with the initial condition $x(t)=x_{0}\left(t \in\left[t_{0}, t_{0}+\tau_{0}\right]\right)$ satisfying (1). We call that $x\left(t, t_{0}, x_{0}\right)$ is a solution of the system (1). If $f(\cdot)$ satisfies that $f(0,0)=0$, and there exist a non-decreasing function $N: \mathbb{R}^{+} \rightarrow \mathbb{R}^{+}$and a positive constant $\lambda$ such that $\left\|x\left(t, t_{0}, x_{0}\right)\right\|<$ $e^{-\lambda\left(t-t_{0}\right)} N\left(\left\|x_{0}\right\|\right)$ for any $x_{0} \in \mathbb{R}^{n}$, then, the system (1) is globally exponentially stable.

Now, we give the following sufficient conditions to ensure the hybrid system (1) is globally exponentially stable.

\section{Theorem 1}

Suppose there exist three positive constants $\rho_{1}, \alpha_{1}, \beta_{1}$, and a positive definite and radially unbounded function $V(x(t))$ defined on $\left[t_{0}, \infty\right)$, satisfying the following conditions

$$
\left.\frac{d V(x(t))}{d t}\right|_{(1)} \leq-\alpha_{1} V(x(t))+\beta_{1} V\left(x\left(t_{k}\right)\right), t \in\left[t_{k}+\tau_{k}, t_{k+1}+\tau_{k+1}\right), k \geq 0,
$$

and

$$
\begin{gathered}
\max _{k \geq 0}\left\{\rho_{1} e^{-\alpha_{1}\left(T_{k+1}-\tau_{k}\right)}+e^{-\alpha_{1}\left(T_{k+1}+\tau_{k+1}-\tau_{k}\right)}\right\}<1, \\
\max _{k \geq 0}\left\{\left(\frac{\beta_{1}}{\alpha_{1}}+\rho_{1} \frac{\beta_{1}}{\alpha_{1}}-\frac{\beta_{1}}{\alpha_{1}} e^{-\alpha_{1}\left(T_{k+1}-\tau_{k}\right)}\left(e^{-\alpha_{1} \tau_{k+1}}+\rho_{1}\right)\right) / \rho_{1}\right\}<1 .
\end{gathered}
$$

Then, the system (1) is globally exponentially stable.

Proof: The main point of the proof is to construct an iteration with a parameter. Then, a convergent sequence with the parameter can be obtained based on the iteration. Therefore, the exponential stability of the system (1) can be derived.

Multiplying the both sides of (2) by $e^{\alpha_{1} t}$ yields

$$
\left.e^{\alpha_{1} t} \frac{d}{d t} V(x(t))\right|_{(1)}+e^{\alpha_{1} t} \alpha_{1} V(x(t)) \leq e^{\alpha_{1} t} \beta_{1} V\left(x\left(t_{k}\right)\right), t \in\left[t_{k}+\tau_{k}, t_{k+1}+\tau_{k+1}\right) .
$$

Integrating the above differential inequality from $t_{k}+\tau_{k}$ to $t$, we have

$$
\begin{gathered}
V(x(t)) \leq e^{-\alpha_{1}\left(t-t_{k}-\tau_{k}\right)} V\left(x\left(t_{k}+\tau_{k}\right)\right)+\frac{\beta_{1}}{\alpha_{1}} V\left(x\left(t_{k}\right)\right) \\
-\frac{\beta_{1}}{\alpha_{1}} e^{-\alpha_{1}\left(t-t_{k}-\tau_{k}\right)} V\left(x\left(t_{k}\right)\right), t \in\left[t_{k}+\tau_{k}, t_{k+1}+\tau_{k+1}\right) .
\end{gathered}
$$

Note that $x(t)$ is continuous on $\left[t_{0}, \infty\right)$. Let $t=t_{k+1}+\tau_{k+1}$ and $t=t_{k+1}$, respectively, then

$$
\begin{gathered}
V\left(x\left(t_{k+1}+\tau_{k+1}\right)\right) \leq e^{-\alpha_{1}\left(T_{k+1}+\tau_{k+1}-\tau_{k}\right)} V\left(x\left(t_{k}+\tau_{k}\right)\right)+\frac{\beta_{1}}{\alpha_{1}} V\left(x\left(t_{k}\right)\right) \\
-\frac{\beta_{1}}{\alpha_{1}} e^{-\alpha_{1}\left(T_{k+1}+\tau_{k+1}-\tau_{k}\right)} V\left(x\left(t_{k}\right)\right),
\end{gathered}
$$

and

$$
V\left(x\left(t_{k+1}\right)\right) \leq e^{-\alpha_{1}\left(T_{k+1}-\tau_{k}\right)} V\left(x\left(t_{k}+\tau_{k}\right)\right)+\frac{\beta_{1}}{\alpha_{1}} V\left(x\left(t_{k}\right)\right)-\frac{\beta_{1}}{\alpha_{1}} e^{-\alpha_{1}\left(T_{k+1}-\tau_{k}\right)} V\left(x\left(t_{k}\right)\right) .
$$

From the above two inequalities, we have

$$
\begin{gathered}
V\left(x\left(t_{k+1}+\tau_{k+1}\right)\right)+\rho_{1} V\left(x\left(t_{k+1}\right)\right) \leq\left(e^{-\alpha_{1}\left(T_{k+1}+\tau_{k+1}-\tau_{k}\right)}+\rho_{1} e^{-\alpha_{1}\left(T_{k+1}-\tau_{k}\right)}\right) V\left(x\left(t_{k}+\tau_{k}\right)\right) \\
+\left(\frac{\beta_{1}}{\alpha_{1}}+\rho_{1} \frac{\beta_{1}}{\alpha_{1}}-\frac{\beta_{1}}{\alpha_{1}} e^{-\alpha_{1}\left(T_{k+1}-\tau_{k}\right)}\left(e^{-\alpha_{1} \tau_{k+1}}+\rho_{1}\right)\right) V\left(x\left(t_{k}\right)\right), \quad k \geq 0 .
\end{gathered}
$$


Let $\quad \eta_{1}=\max \left\{\max _{k \geq 0}\left\{\rho_{1} e^{-\alpha_{1}\left(T_{k+1}-\tau_{k}\right)}+e^{-\alpha_{1}\left(T_{k+1}+\tau_{k+1}-\tau_{k}\right)}\right\}, \quad \max _{k \geq 0}\left\{\left(\frac{\beta_{1}}{\alpha_{1}}+\rho_{1} \frac{\beta_{1}}{\alpha_{1}}-\right.\right.\right.$ $\left.\left.\left.\frac{\beta_{1}}{\alpha_{1}} e^{-\alpha_{1}\left(T_{k+1}-\tau_{k}\right)}\left(e^{-\alpha_{1} \tau_{k+1}}+\rho_{1}\right)\right) / \rho_{1}\right\}\right\}$. From (3), we have $0<\eta_{1}<1$. Therefore, it follows from (5) that

$$
V\left(x\left(t_{k+1}+\tau_{k+1}\right)\right)+\rho_{1} V\left(x\left(t_{k+1}\right)\right) \leq \eta_{1}\left[V\left(x\left(t_{k}+\tau_{k}\right)\right)+\rho_{1} V\left(x\left(t_{k}\right)\right)\right], \quad k \geq 0 .
$$

Applying iteratively (6) with the parameter $\rho_{1}$, we have

$$
V\left(x\left(t_{k}+\tau_{k}\right)\right)+\rho_{1} V\left(x\left(t_{k}\right)\right) \leq \eta_{1}^{k}\left[V\left(x\left(t_{0}+\tau_{0}\right)\right)+\rho_{1} V\left(x\left(t_{0}\right)\right)\right], \quad k \geq 0,
$$

that is, the sequence $\left\{V\left(x\left(t_{k}+\tau_{k}\right)\right)+\rho_{1} V\left(x\left(t_{k}\right)\right)\right\}$ is convergent. It follows from (4) and (7) that

$$
\begin{gathered}
V(x(t)) \leq V\left(x\left(t_{k}+\tau_{k}\right)\right)+\frac{\beta_{1}}{\alpha_{1}} V\left(x\left(t_{k}\right)\right) \leq \eta_{1}^{k}\left(1+\frac{\beta_{1}}{\alpha_{1} \rho_{1}}\right)\left[V\left(x\left(t_{0}+\tau_{0}\right)\right)+\rho_{1} V\left(x\left(t_{0}\right)\right)\right], \\
t \in\left[t_{k}+\tau_{k}, t_{k+1}+\tau_{k+1}\right) .
\end{gathered}
$$

For any $t>t_{0}+\tau_{0}$, there exists $k \geq 0$ such that $t \in\left[t_{k}+\tau_{k}, t_{k+1}+\tau_{k+1}\right)$. Then, $\frac{t-t_{0}-\tau_{0}}{T_{\max }+\tau}-1 \leq k$. Therefore,

$$
\begin{aligned}
& V(x(t)) \leq \eta_{1}^{\frac{t-t_{0}-\tau_{0}}{T_{\max }+\tau}-1}\left(1+\frac{\beta_{1}}{\alpha_{1} \rho_{1}}\right)\left[V\left(x\left(t_{0}+\tau_{0}\right)\right)+\rho_{1} V\left(x\left(t_{0}\right)\right)\right. \\
= & e^{\frac{t}{T_{\max }+\tau} \ln \eta_{1}} \eta_{1}^{\frac{-t_{0}-\tau_{0}}{T_{\max }+\tau}-1}\left(1+\frac{\beta_{1}}{\alpha_{1} \rho_{1}}\right)\left[V\left(x\left(t_{0}+\tau_{0}\right)\right)+\rho_{1} V\left(x\left(t_{0}\right)\right)\right],
\end{aligned}
$$

which implies that the system (1) is globally exponentially stable.

For Theorem 1, we have the following corollaries.

\section{Corollary 1}

If there exist three positive constants $\rho_{1}, \alpha_{1}, \beta_{1}$ and a positive definite and radially unbounded function $V(x(t))$ defined on $\left[t_{0}, \infty\right)$, such that the condition (2) and

$$
e^{-\alpha_{1}\left(T_{\min }-\tau\right)}\left(1+\rho_{1}\right)<1, \frac{\beta_{1}\left(1+\rho_{1}\right)}{\alpha_{1} \rho_{1}}<1,
$$

hold, then, the system (1) is globally exponentially stable.

\section{Corollary 2}

If there exist two positive constants $\alpha_{1}, \beta_{1}$ and a positive definite and radially unbounded function $V(x(t))$ defined on $\left[t_{0}, \infty\right)$, satisfying the condition (2) and

$$
\alpha_{1}>\beta_{1}, T_{\min }-\tau>\frac{\beta_{1}}{\alpha_{1}\left(\alpha_{1}-\beta_{1}\right)},
$$

then, the system (1) is globally exponentially stable.

Proof: The condition (9) implies that there exists $\rho>0$ such that $e^{\alpha_{1}\left(T_{\min }-\tau\right)}-1>\alpha_{1}\left(T_{\min }-\right.$ $\tau)>\rho>\frac{\beta_{1}}{\alpha_{1}-\beta_{1}}$. Therefore, the conditions (8) hold. The proof is completed.

\section{Remark 2}

The condition (9) can hold for sufficiently large value of $T_{\min }$, that is, there is no upper bound of $T_{k}+\tau_{k}$. In fact, the inequality (4) holds on $\left[t_{k}+\tau_{k}, t_{k+1}+\tau_{k+1}\right]$. If $T_{k}$ is sufficiently large, we have

$$
V\left(x\left(t_{k+1}+\tau_{k+1}\right)\right)<\frac{\beta_{1}}{\alpha_{1}} V\left(x\left(t_{k}+\tau_{k}\right)\right) .
$$

Then,

$$
V\left(x\left(t_{k}+\tau_{k}\right)\right)<\left(\frac{\beta_{1}}{\alpha_{1}}\right)^{k} V\left(x\left(t_{0}+\tau_{0}\right)\right)<\left(\frac{\rho_{1}}{1+\rho_{1}}\right)^{k} V\left(x\left(t_{0}+\tau_{0}\right)\right),
$$

which implies that the system (1) is globally exponential stable. 


\section{Continuous output feedback stabilization for a class of nonlinear systems with sampled and delayed measurements}

In this section, our aim is to propose an output feedback stabilization for the following system

$$
\left\{\begin{array}{l}
\dot{x}_{1}(t)=x_{2}(t)+f_{1}\left(x_{1}(t)\right) \\
\quad \vdots \\
\dot{x}_{n-1}(t)=x_{n}(t)+f_{n-1}\left(x_{1}(t), x_{2}(t), \cdots, x_{n-1}(t)\right), \\
\dot{x}_{n}(t)=f_{n}\left(x_{1}(t), x_{2}(t), \cdots, x_{n}(t)\right)+u(t), \\
y(t)=x_{1}(t),
\end{array}\right.
$$

where $x(t) \in \mathbb{R}^{n}$ is the state, $u(t) \in \mathbb{R}$ is the input. We make the following assumptions. The output $y(t)$ is sampled at instants $t_{k}$ and is available at instants $t_{k}+\tau_{k}$, where $\left\{t_{k}\right\}$ is a strictly increasing sequence and satisfies $\lim _{k \rightarrow \infty} t_{k}=\infty$, and $\tau_{k}>0$ represents the transmission delay. The sampling interval $T_{k+1}=t_{k+1}-t_{k}$ satisfies $0<T_{\min } \leq t_{k+1}-t_{k} \leq T_{\max }$ for two positive real numbers $T_{\min }, T_{\max }$ and for all $k=0,1, \cdots, \infty$. The transmission delays $\tau_{k}$ are unknown, but have an upper bound $\tau$. We also assume that $\tau \leq T_{\min }$, that is, the measures sampled at instants $t_{k}$ are available for the observer before the next measures sampled at instants $t_{k+1}$. We assume that $f_{i}\left(x_{1}, \cdots, x_{i}\right)$ $(i=1 \cdots, n)$ are unknown and satisfy the following conditions

$$
\left|f_{i}\left(x_{1}, \cdots, x_{i}\right)\right| \leq l_{1}\left(\left|x_{1}\right|+\cdots+\left|x_{i}\right|\right), i=1, \cdots, n,
$$

where $l_{1}$ is a positive real number.

\section{Remark 3}

The system (10) is firstly introduced in [28], where a linear state feedback control law has been proposed to achieve global exponential stability without considering sampled measurements. Under the same condition, the authors in [30] have presented a linear dynamic output compensator to globally exponentially stabilize the system (10). In [29], an exponential observer has been built for a biological system. Global asymptotic stabilization via output feedback has also been studied for nonlinear systems similar to (10) in [1]. The dynamics considered in [1] are in a feedback form and the nonlinear terms have an incremental rate which depends on the measured output. In $[17,19]$, a state feedback law has been constructed to achieve global asymptotic stabilization for the nonlinear system (10) under sampled and delayed measurements, and with inputs subject to delay and zeroorder hold.

Next, we construct the following output feedback stabilizer

$$
\begin{aligned}
& \left\{\begin{array}{l}
\dot{\hat{x}}_{1}(t)=\hat{x}_{2}(t)+L a_{1} e_{1}\left(t_{k}\right), \\
\quad \vdots \\
\dot{\hat{x}}_{n-1}(t)=\hat{x}_{n}(t)+L^{n-1} a_{n-1} e_{1}\left(t_{k}\right), \\
\dot{\hat{x}}_{n}(t)=L^{n} a_{n} e_{1}\left(t_{k}\right)+u(t), \\
\hat{x}_{i}\left(t_{k+1}+\tau_{k+1}\right)=\lim _{t \rightarrow\left(t_{k+1}+\tau_{k+1}\right)^{-}} \hat{x}_{i}(t), \\
i=1, \cdots, n, \quad t \in\left[t_{k}+\tau_{k}, t_{k+1}+\tau_{k+1}\right), \quad k \geq 0,
\end{array}\right. \\
& u(t)=-\left[L^{n} k_{1} \hat{x}_{1}(t)+L^{n-1} k_{2} \hat{x}_{2}(t)+\cdots+L k_{n} \hat{x}_{n}(t)\right],
\end{aligned}
$$

where $\hat{x}(t)=\hat{x}\left(t_{0}\right)=\hat{x}_{0}$ for $t \in\left[t_{0}-T_{\max }-\tau, t_{0}+\tau_{0}\right]$ and $L \geq 1, e_{1}\left(t_{k}\right)=x_{1}\left(t_{k}\right)-\hat{x}_{1}\left(t_{k}\right)$, $a_{i}>0$ and $k_{i}>0, i=1,2, \cdots, n$ are the coefficients of the Hurwitz polynomial $s^{n}+w_{1} s^{n-1}+$ $\cdots+w_{n-1} s+w_{n}$, with $w_{i}=a_{i}$ or $w_{i}=k_{n-i+1}$. Now, we give the definition of output feedback stabilization for the system (10).

\section{Definition 2}

We call that the $n$-dimensional system (10) is globally exponentially stabilizable under the condition (11) and $x(t)=x_{0}$ for $t \in\left[t_{0}-T_{\max }-\tau, t_{0}\right]$, if there exists an $n$-dimensional dynamical system (12) such that the $2 n$-dimensional subsystem (10)-(12) satisfies $\|\hat{x}(t)\| \leq$ 
$e^{-\lambda\left(t-t_{0}\right)} N\left(\left\|\hat{x}_{0}\right\|,\left\|x_{0}\right\|\right)$ and $\|\hat{x}(t)-x(t)\| \leq e^{-\lambda\left(t-t_{0}\right)} N\left(\left\|\hat{x}_{0}\right\|,\left\|x_{0}\right\|\right)$ for any $x_{0} \in \mathbb{R}^{n}$ and $\hat{x}_{0} \in$ $\mathbb{R}^{n}$, where $\lambda>0$ and $N: \mathbb{R}^{+} \rightarrow \mathbb{R}^{+}$is a non-decreasing function. Or, we call that $n$-dimensional dynamical system (12) globally exponentially stabilizes the $n$-dimensional system (10) under the condition (11).

The following lemma is also useful for our main results.

\section{Lemma 1}

[25] For any positive definite matrix $U \in \mathbb{R}^{n \times n}$, scalar $\gamma>0$, vector function $w:[0, \gamma] \rightarrow \mathbb{R}^{n}$ such that the integrations concerned are well defined, the following inequality holds

$$
\left[\int_{0}^{\gamma} w(s) d s\right]^{T} U\left[\int_{0}^{\gamma} w(s) d s\right] \leq \gamma\left[\int_{0}^{\gamma} w(s)^{T} U w(s) d s\right] .
$$

Let $e_{i}(t)=x_{i}(t)-\hat{x}_{i}(t)$ denote the estimation error of the high gain observer (12). Then, the error dynamics is given by

$$
\left\{\begin{array}{c}
\dot{e}_{1}(t)=e_{2}(t)-L a_{1} e_{1}\left(t_{k}\right)+f_{1}\left(x_{1}(t)\right) \\
\vdots \\
\dot{e}_{n-1}(t)=e_{n}(t)-L^{n-1} a_{n-1} e_{1}\left(t_{k}\right) \\
\quad+f_{n-1}\left(x_{1}(t), x_{2}(t), \cdots, x_{n-1}(t)\right), \\
\dot{e}_{n}(t)=-L^{n} a_{n} e_{1}\left(t_{k}\right)+f_{n}\left(x_{1}(t), x_{2}(t), \cdots, x_{n}(t)\right) \\
e_{i}\left(t_{k+1}+\tau_{k+1}\right)=\lim _{t \rightarrow\left(t_{k+1}+\tau_{k+1}\right)-e(t),} \\
i=1, \cdots, n, t \in\left[t_{k}+\tau_{k}, t_{k+1}+\tau_{k+1}\right), k \geq 0
\end{array}\right.
$$

In order to simplify the analysis, we consider the following coordinate transformation

$$
\varepsilon_{i}(t)=\frac{e_{i}(t)}{L^{i}}, z_{i}(t)=\frac{\hat{x}_{i}(t)}{L^{i}}, i=1,2, \cdots, n .
$$

The closed-loop system (10) and (14) can be expressed as

$$
\left\{\begin{array}{l}
\dot{\varepsilon}_{1}(t)=L \varepsilon_{2}(t)-L a_{1} \varepsilon_{1}(t)+L a_{1}\left(\varepsilon_{1}(t)-\varepsilon_{1}\left(t_{k}\right)\right)+\frac{f_{1}}{L}, \\
\quad \vdots \\
\dot{\varepsilon}_{n-1}(t)=L \varepsilon_{n}(t)-L a_{n-1} \varepsilon_{1}(t)+L a_{n-1}\left(\varepsilon_{1}(t)-\varepsilon_{1}\left(t_{k}\right)\right)+\frac{f_{n-1}}{L^{n-1}}, \\
\dot{\varepsilon}_{n}(t)=-L a_{n} \varepsilon_{1}(t)+L a_{n}\left(\varepsilon_{1}(t)-\varepsilon_{1}\left(t_{k}\right)\right)+\frac{f_{n}}{L^{n}} \\
\varepsilon_{i}\left(t_{k+1}+\tau_{k+1}\right)=\lim _{t \rightarrow\left(t_{k+1}+\tau_{k+1}\right)-\varepsilon_{i}(t), i=1, \cdots, n}
\end{array}\right.
$$

and

$$
\left\{\begin{array}{l}
\dot{z}_{1}(t)=L z_{2}(t)+L a_{1} \varepsilon_{1}(t)-L a_{1}\left(\varepsilon_{1}(t)-\varepsilon_{1}\left(t_{k}\right)\right), \\
\quad \vdots \\
\dot{z}_{n-1}(t)=L z_{n}(t)+L a_{n-1} \varepsilon_{1}(t)-L a_{n-1}\left(\varepsilon_{1}(t)-\varepsilon_{1}\left(t_{k}\right)\right), \\
\dot{z}_{n}(t)=-L\left(k_{1} z_{1}(t)+\cdots+k_{n} z_{n}(t)\right)+L a_{n} \varepsilon_{1}(t)-L a_{n}\left(\varepsilon_{1}(t)-\varepsilon_{1}\left(t_{k}\right)\right), \\
z_{i}\left(t_{k+1}+\tau_{k+1}\right)=\lim _{t \rightarrow\left(t_{k+1}+\tau_{k+1}\right)-z_{i}(t),} \\
\quad i=1, \cdots, n, t \in\left[t_{k}+\tau_{k}, t_{k+1}+\tau_{k+1}\right), k \geq 0 .
\end{array}\right.
$$

Now, we give the following results.

\section{Theorem 2}

There exists an output feedback control law in the form of (12) which globally exponentially stabilizes the system (10) with the condition (11), if $a_{i}>0$ and $k_{i}>0(i=1, \cdots, n)$ are selected such that there exist two symmetric positive definite matrices $P$ and $Q$ such that

$$
\begin{gathered}
A^{T} P+P A \leq-I, \\
B^{T} Q+Q B \leq-2 I,
\end{gathered}
$$


are satisfied, and $L$ satisfies

$$
L>\left\{1, l_{1}, 6 n l_{1} \lambda_{1}, 2\left(\beta_{2}+1\right) n l_{1} \lambda_{1}\right\}
$$

and

$$
T_{\max }+\tau \leq \frac{1}{c_{3} L}, T_{\min }-\tau>\left(\frac{c_{2}}{c_{1}-c_{2}}\right) \frac{1}{c_{1} L},
$$

where $c_{1}=\min \left\{\frac{1}{8 \lambda_{1}\left(\beta_{2}+1\right)}, \frac{1}{8 \lambda_{3}}\right\}, c_{3}>\max \left\{8,16 n \bar{a}_{1}\left(\lambda_{1}^{2}\left(\beta_{2}+1\right)+\lambda_{3}^{2}\right)+c_{1}, \frac{\kappa_{1}}{c_{1}}, \frac{\kappa_{1}+\sqrt{\kappa_{1}^{2}+4 c_{1}^{3} \kappa_{1}}}{2 c_{1}^{2}}\right\}$, $c_{2}=\frac{\kappa_{1}}{c_{3}^{2}}, \quad \kappa_{1}=\frac{16 n \bar{a}_{1}^{2}\left(\lambda_{1}^{2}\left(\beta_{2}+1\right)+\lambda_{3}^{2}\right)}{\lambda_{2}\left(\beta_{2}+1\right)}, \quad$ and $\quad \bar{a}_{1}=\max \left\{a_{i}^{2}\right\}, \quad \lambda_{1}=\lambda_{\max }(P), \quad \lambda_{2}=\lambda_{\min }(P)$, $\lambda_{3}=\lambda_{\max }(Q), \quad \lambda_{4}=\lambda_{\min }(Q), \quad \beta_{2}=4 n \bar{a}_{1} \lambda_{3}^{2}, \quad A=\left[\begin{array}{cccc}-a_{1} & 1 & \cdots & 0 \\ \vdots & \vdots & \ddots & \vdots \\ -a_{n-1} & 0 & \cdots & 1 \\ -a_{n} & 0 & \cdots & 0\end{array}\right], \quad B=$ $\left[\begin{array}{cccc}0 & 1 & \cdots & 0 \\ \vdots & \vdots & \ddots & \vdots \\ 0 & 0 & \cdots & 1 \\ -k_{1} & -k_{2} & \cdots & -k_{n}\end{array}\right]$

Proof: Consider the following positive definite function

$$
V_{1}(t)=\left(\beta_{2}+1\right) \varepsilon(t)^{T} P \varepsilon(t), \quad V_{2}(t)=z(t)^{T} Q z(t),
$$

where $\varepsilon(t)=\left[\varepsilon_{1}(t), \cdots, \varepsilon_{n}(t)\right]^{T}, z(t)=\left[z_{1}(t), \cdots, z_{n}(t)\right]^{T}$. Let $F=\left(\frac{f_{1}}{L}, \frac{f_{2}}{L^{2}}, \cdots, \frac{f_{n}}{L^{n}}\right)^{T}$. Then,

$$
\begin{gathered}
\left.\frac{d V_{1}(t)}{d t}\right|_{(15)}+\left.\frac{d V_{2}(t)}{d t}\right|_{(16)}=L\left(\beta_{2}+1\right) \varepsilon(t)^{T}\left(P A+A^{T} P\right) \varepsilon(t)+2\left(\beta_{2}+1\right) \varepsilon(t)^{T} P F \\
+2 L\left(\beta_{2}+1\right) \varepsilon(t)^{T} P\left[a_{1}, \cdots, a_{n}\right]^{T}\left(\varepsilon_{1}(t)-\varepsilon_{1}\left(t_{k}\right)\right)+L z(t)^{T}\left(Q B+B^{T} Q\right) z(t) \\
+2 L z(t)^{T} Q\left[a_{1}, \cdots, a_{n}\right]^{T} \varepsilon_{1}(t)-2 L z(t)^{T} Q\left[a_{1}, \cdots, a_{n}\right]^{T}\left(\varepsilon_{1}(t)-\varepsilon_{1}\left(t_{k}\right)\right) \\
\leq-L\left(\beta_{2}+1\right) \varepsilon(t)^{T} \varepsilon(t)+\frac{1}{4} L\left(\beta_{2}+1\right) \varepsilon(t)^{T} \varepsilon(t)+4 L\left(\beta_{2}+1\right) \lambda_{1}^{2} n \bar{a}_{1}\left(\varepsilon_{1}(t)-\varepsilon_{1}\left(t_{k}\right)\right)^{2} \\
+2\left(\beta_{2}+1\right) \varepsilon(t)^{T} P F-2 L z(t)^{T} z(t)+L z(t)^{T} z(t)+L n \lambda_{3}^{2} \bar{a}_{1} \varepsilon_{1}(t)^{2}+\frac{1}{4} L z(t)^{T} z(t) \\
+4 L n \bar{a}_{1} \lambda_{3}^{2}\left(\varepsilon_{1}(t)-\varepsilon_{1}\left(t_{k}\right)\right)^{2} \\
\leq-\frac{3}{4} L\left(\beta_{2}+1\right) \varepsilon(t)^{T} \varepsilon(t)+4 L\left(\beta_{2}+1\right) \lambda_{1}^{2} n \bar{a}_{1}\left[\varepsilon_{1}(t)-\varepsilon_{1}\left(t_{k}\right)\right]^{2}+2\left(\beta_{2}+1\right) \varepsilon(t)^{T} P F \\
-\frac{3}{4} L z(t)^{T} z(t)+L n \lambda_{3}^{2} \bar{a}_{1} \varepsilon_{1}^{2}(t)+4 L n \bar{a}_{1} \lambda_{3}^{2}\left[\varepsilon_{1}(t)-\varepsilon_{1}\left(t_{k}\right)\right]^{2}, t \in\left[t_{k}+\tau_{k}, t_{k+1}+\tau_{k+1}\right) .
\end{gathered}
$$

In addition, from the condition (11), it follows that

$$
\begin{aligned}
& \left|2 \varepsilon(t)^{T} P F\right| \leq 2\left(\varepsilon^{T}(t) P \varepsilon(t)\right)^{1 / 2}\left(F^{T} P F\right)^{1 / 2} \leq 2 n l_{1} \lambda_{1}\left(\varepsilon^{T}(t) \varepsilon(t)\right)^{1 / 2} \\
& \times\left[\left(\varepsilon^{T}(t) \varepsilon(t)\right)^{1 / 2}+\left(z(t)^{T} z(t)\right)^{1 / 2}\right] \leq n l_{1} \lambda_{1}\left(3 \varepsilon^{T}(t) \varepsilon(t)+z^{T}(t) z(t)\right) .
\end{aligned}
$$

By Lemma 1, we have

$$
\begin{gathered}
\left|\varepsilon_{1}(t)-\varepsilon_{1}\left(t_{k}\right)\right|^{2} \leq\left(t-t_{k}\right) \int_{t_{k}}^{t}\left|\dot{\varepsilon}_{1}(s)\right|^{2} d s \\
\leq 4\left(t-t_{k}\right) L^{2} \int_{t_{k}}^{t}\left[\varepsilon_{2}(s)^{2}+\frac{l_{1}^{2}}{L^{2}}\left(z_{1}(s)^{2}+\varepsilon_{1}(s)^{2}\right)+\bar{a}_{1} \varepsilon_{1}\left(t_{k}\right)^{2}\right] d s, t \in\left[t_{k}+\tau_{k}, t_{k+1}+\tau_{k+1}\right) .
\end{gathered}
$$

From (22), (23) and (24), it follows that

$$
\begin{gathered}
\left.\quad \frac{d V_{1}(t)}{d t}\right|_{(15)}+\left.\frac{d V_{2}(t)}{d t}\right|_{(16)} \leq-\left(\beta_{2}+1\right)\left[\frac{3}{4} L-3 n l_{1} \lambda_{1}\right] \varepsilon(t)^{T} \varepsilon(t)+16\left(\beta_{2}+1\right) L^{3} \\
\times n \bar{a}_{1}^{2} \lambda_{1}^{2}\left(t-t_{k}\right)^{2} \varepsilon_{1}\left(t_{k}\right)^{2}+16\left(\beta_{2}+1\right) L^{3} n \bar{a}_{1} \lambda_{1}^{2}\left(t-t_{k}\right) \int_{t_{k}}^{t}\left[\varepsilon_{1}(s)^{2}+\varepsilon_{2}(s)^{2}+z_{1}(s)^{2}\right] d s \\
-\left(\frac{3}{4} L-\left(\beta_{2}+1\right) n l_{1} \lambda_{1}\right) z(t)^{T} z(t)+L n \lambda_{3}^{2} \bar{a}_{1} \varepsilon(t)^{T} \varepsilon(t)+16 L^{3} n \bar{a}_{1}^{2} \lambda_{3}^{2}\left(t-t_{k}\right)^{2} \varepsilon_{1}\left(t_{k}\right)^{2} \\
+16 L^{3} n \bar{a}_{1} \lambda_{3}^{2}\left(t-t_{k}\right) \int_{t_{k}}^{t}\left[\varepsilon_{1}(s)^{2}+\varepsilon_{2}(s)^{2}+z_{1}(s)^{2}\right] d s, \quad t \in\left[t_{k}+\tau_{k}, t_{k+1}+\tau_{k+1}\right) .
\end{gathered}
$$

Construct the following auxiliary integral function:

$$
V_{3}(t)=\int_{t-T_{\max }-\tau}^{t} \int_{\rho}^{t}\left[\varepsilon(s)^{T} \varepsilon(s)+z(s)^{T} z(s)\right] d s d \rho, t \in\left[t_{0}, \infty\right) .
$$


We have

$$
\frac{d V_{3}(t)}{d t}=\left(T_{\max }+\tau\right)\left(\varepsilon(t)^{T} \varepsilon(t)+z(t)^{T} z(t)\right)-\int_{t-T_{\max }-\tau}^{t}\left(\varepsilon(s)^{T} \varepsilon(s)+z(s)^{T} z(s)\right) d s,
$$

and

$$
V_{3}(t) \leq\left(T_{\max }+\tau\right) \int_{t-T_{\max }-\tau}^{t}\left[\varepsilon(s)^{T} \varepsilon(s)+z(s)^{T} z(s)\right] d s, t \in\left[t_{0}, \infty\right) .
$$

Now, consider the following Lyapunov-Krasovskii function

$$
V(t)=V_{1}(t)+V_{2}(t)+L^{2} V_{3}(t) .
$$

From (19), (25), (25) and (26), we can obtain

$$
\begin{gathered}
\left.\frac{d V(t)}{d t}\right|_{(15),(16)} \leq-\left[\left(\beta_{2}+1\right)\left(\frac{3}{4} L-3 n l_{1} \lambda_{1}\right)-n \lambda_{3}^{2} \bar{a}_{1} L\right] \varepsilon(t)^{T} \varepsilon(t)-\left[\frac{3}{4} L-\left(\beta_{2}+1\right) n l_{1} \lambda_{1}\right] z(t)^{T} z(t) \\
+\left(T_{\max }+\tau\right) L^{2}\left[\varepsilon(t)^{T} \varepsilon(t)+z(t)^{T} z(t)\right]+\left[16 L^{3} n \bar{a}_{1}\left(\lambda_{1}^{2}\left(\beta_{2}+1\right)+\lambda_{3}^{2}\right)\left(t-t_{k}\right)-L^{2}\right] \\
\int_{t-T_{\max }-\tau}^{t}\left[\varepsilon(s)^{T} \varepsilon(s)+z(s)^{T} z(s)\right] d s+16 L^{3} n \bar{a}_{1}^{2}\left(\lambda_{1}^{2}\left(\beta_{2}+1\right)+\lambda_{3}^{2}\right)\left(t-t_{k}\right)^{2} \varepsilon_{1}\left(t_{k}\right)^{2} \\
\leq-\frac{1}{\lambda_{1}}\left[\frac{1}{4}-\left(T_{\max }+\tau\right) L\right] L V_{1}(t)+\left[16 n \bar{a}_{1}\left(\lambda_{1}^{2}\left(\beta_{2}+1\right)+\lambda_{3}^{2}\right)-\frac{1}{\left(T_{\max }+\tau\right) L}\right] L^{3} V_{3}(t) \\
\quad-\frac{1}{\lambda_{3}}\left[\left(\frac{1}{4}-\left(T_{\max }+\tau\right) L\right] L V_{2}(t)+\frac{16 L^{3} n \bar{a}_{1}^{2}\left(\lambda_{1}^{2}\left(\beta_{2}+1\right)+\lambda_{3}^{2}\right)}{\lambda_{2}\left(\beta_{2}+1\right)}\left(T_{\max }+\tau\right)^{2} V_{1}\left(t_{k}\right) .\right.
\end{gathered}
$$

Note that $L>\left\{6 n l_{1} \lambda_{1}, 2\left(\beta_{2}+1\right) n l_{1} \lambda_{1}\right\}, \beta_{2}=4 n \lambda_{3}^{2} \bar{a}_{1}, T_{\max }+\tau<\frac{1}{c_{3} L}$, then, we have

$$
\begin{aligned}
\left.\frac{d V(t)}{d t}\right|_{(15),(16) \leq} & -\frac{1}{\lambda_{1}\left(\beta_{2}+1\right)}\left(\frac{1}{4}-\frac{1}{c_{3}}\right) L V_{1}(t)+\left[16 n \bar{a}_{1}\left(\lambda_{1}^{2}\left(\beta_{2}+1\right)+\lambda_{3}^{2}\right)-c_{3}\right] L^{3} V_{3}(t) \\
& -\frac{1}{\lambda_{3}}\left(\frac{1}{4}-\frac{1}{c_{3}}\right) L V_{2}(t)+\frac{16 n \bar{a}_{1}^{2}\left(\lambda_{1}^{2}\left(\beta_{2}+1\right)+\lambda_{3}^{2}\right)}{\lambda_{2}\left(\beta_{2}+1\right) c_{3}^{2}} L V_{1}\left(t_{k}\right) .
\end{aligned}
$$

Since $c_{3}>\max \left\{8,16 n \bar{a}_{1}\left(\lambda_{1}^{2}\left(\beta_{2}+1\right)+\lambda_{3}^{2}\right)+c_{1}, \frac{\kappa_{1}}{c_{1}}, \frac{\kappa_{1}+\sqrt{\kappa_{1}^{2}+4 c_{1}^{3} \kappa_{1}}}{2 c_{1}^{2}}\right\}, c_{1}=\min \left\{\frac{1}{8 \lambda_{1}\left(\beta_{2}+1\right)}, \frac{1}{8 \lambda_{3}}\right\}$, $c_{2}=\frac{\kappa_{1}}{c_{3}^{2}}$, then,

$$
\begin{aligned}
& \left.\frac{d V(t)}{d t}\right|_{(15),(16)} \leq-c_{1} L V_{1}(t)-c_{1} L^{3} V_{3}(t)-c_{1} L V_{2}(t)+c_{2} L V_{1}\left(t_{k}\right) \\
& \quad \leq-c_{1} L V(t)+c_{2} L V\left(t_{k}\right), t \in\left[t_{k}+\tau_{k}, t_{k+1}+\tau_{k+1}\right), k \geq 0 .
\end{aligned}
$$

Let $\alpha_{1}=c_{1} L, \beta_{1}=c_{2} L$, and $\left(\varepsilon\left(t, t_{0}, \varepsilon_{0}, z_{0}\right), z\left(t, t_{0}, \varepsilon_{0}, z_{0}\right)\right)$ denote the solution of (15)(16). Then, $T_{\min }-\tau>\frac{c_{2}}{c_{1}\left(c_{1}-c_{2}\right) L}$ implies that there exists $\rho_{1}>0$ such that $\eta_{1}=$ $\max \left\{e^{-\alpha_{1}\left(T_{\min }-\tau\right)}\left(1+\rho_{1}\right), \frac{\beta_{1}\left(1+\rho_{1}\right)}{\alpha_{1} \rho_{1}}\right\}<1$. Using the same methods as in Theorem 1, we obtain that $\quad V(\varepsilon(t)) \leq e^{\frac{t}{T_{\max }+\tau} \ln \eta_{1}} \eta_{1}^{\frac{-t_{0}-\tau_{0}}{T_{\max +\tau}}-1}\left[V\left(\varepsilon\left(t_{0}+\tau_{0}, t_{0}, \varepsilon_{0}, z_{0}\right)+\rho_{1} V\left(\varepsilon\left(t_{0}, t_{0}, \varepsilon_{0}, z_{0}\right)\right)\right], t \geq t_{0}\right.$. Then, $\|\hat{x}(t)-x(t)\| \leq e^{\frac{t}{T_{\max }+\tau} \ln \eta_{1}} N_{1} \quad$ and $\|\hat{x}(t)\| \leq e^{\frac{t}{T_{\max }+\tau} \ln \eta_{1}} N_{2}, t \geq t_{0}$, where $N_{1}=$ $\frac{L^{2(n-1)}}{\left(\beta_{2}+1\right) \lambda_{2}} \quad \eta_{1}^{\frac{-t_{0}-\tau_{0}-1}{T_{\max }+\tau}-1}\left[V\left(\varepsilon\left(t_{0}+\tau_{0}, t_{0}, \varepsilon_{0}, z_{0}\right) \quad+\rho_{1} \quad V\left(\varepsilon\left(t_{0}, t_{0}, \varepsilon_{0}, \quad z_{0}\right)\right)\right] \quad\right.$ and $\quad N_{2}=\frac{L^{2(n-1)}}{\lambda_{4}}$ $\eta_{1}^{\frac{-t_{0}-\tau_{0}}{T_{\max }+\tau}-1}\left[V\left(\varepsilon\left(t_{0}+\tau_{0}, t_{0}, \varepsilon_{0}, z_{0}\right)+\rho_{1} V\left(\varepsilon\left(t_{0}, t_{0}, \varepsilon_{0}, z_{0}\right)\right)\right]\right.$.

Next, we will prove that any solutions of (10) don't finite-time escape on the interval $\left[t_{0}, t_{0}+\tau_{0}\right] . \quad$ In fact, from (13), we have $u(t)=u_{0}=-\left[L^{n} k_{1} \hat{x}_{1}\left(t_{0}\right)+L^{n-1} k_{2} \hat{x}_{2}\left(t_{0}\right)+\right.$ $\left.\cdots+L k_{n} \hat{x}_{n}\left(t_{0}\right)\right] \quad$ for $\quad t \in\left[t_{0}, t_{0}+\tau_{0}\right]$. Let $\tilde{f}(x(t))=\left[x_{2}(t)+f_{1}\left(x_{1}(t)\right), \cdots, x_{n}(t)+\right.$ $\left.f_{n-1}\left(x_{1}(t), \cdots, x_{n-1}(t)\right), f_{n}\left(x_{1}(t), \cdots, x_{n}(t)\right)\right]^{T}+\tilde{u}$, where $\tilde{u}=\left[0, \cdots, 0, u_{0}\right]^{T}$. Then, the system (10) with the control $u(t)=u_{0}$ on $\left[t_{0}, t_{0}+\tau_{0}\right]$ can be expressed as

$$
\dot{x}(t)=\tilde{f}(x(t))+\tilde{u}, t \in\left[t_{0}, t_{0}+\tau_{0}\right] .
$$

Integrating the differential equation (28) from $t_{0}$ to $t$ yields,

$$
x(t)=x_{0}+\int_{t_{0}}^{t}(\tilde{f}(x(s))+\tilde{u}) d s, t \in\left[t_{0}, t_{0}+\tau_{0}\right] .
$$


Moreover, it follows from (11) that there exists a constant $l_{2}>0$ such that $\|\tilde{f}(x(t))\| \leq l_{2}\|x(t)\|$. Therefore,

$$
\|x(t)\| \leq\left\|x_{0}\right\|+\int_{t_{0}}^{t}\left(\|\tilde{f}(x(s))\|+\left|u_{0}\right|\right) d s \leq\left\|x_{0}\right\|+\int_{t_{0}}^{t}\left(l_{2}\|x(s)\|+\left|u_{0}\right|\right) d s, t \in\left[t_{0}, t_{0}+\tau_{0}\right] .
$$

By Gronwell Lemma [31], we have

$$
\|x(t)\| \leq\left(\left\|x_{0}\right\|+\tau_{0}\left|u_{0}\right|\right) e^{l_{2} \tau_{0}}, t \in\left[t_{0}, t_{0}+\tau_{0}\right] .
$$

The proof is completed.

From Theorem 2, we also have the following results.

\section{Corollary 3}

There exists an output feedback control law in the form of (12) which globally exponentially stabilizes the system (10) with the condition (11), if $a_{i}>0$ and $k_{i}>0(i=1, \cdots, n)$ are selected such that there exist two symmetric positive definite matrices $P, Q$ and a constant $L \geq 1$ such that (17), (18), (19) and

$$
\tau<\frac{1}{\rho_{2} L}\left(\frac{1}{c_{3}}-\frac{1}{c_{4}}\right), T_{\max }<\frac{1}{c_{3} L}-\frac{1}{\rho_{2} L}\left(\frac{1}{c_{3}}-\frac{1}{c_{4}}\right), T_{\min }>\frac{1}{c_{4} L}+\frac{1}{\rho_{2} L}\left(\frac{1}{c_{3}}-\frac{1}{c_{4}}\right),
$$

are satisfied, where $\rho_{2}>2, c_{4}=\frac{\left(c_{1}-c_{2}\right) c_{1}}{c_{2}}, c_{1}, c_{3}, c_{2}$ and $\kappa_{1}$ are given in Theorem 2 .

Proof: Note that $c_{2}=\frac{\kappa_{1}}{c_{3}^{2}}$. Then $c_{3}>\frac{\kappa_{1}}{c_{1}}$ implies that $c_{1}>\frac{\kappa_{1}}{c_{3}}=\frac{\kappa_{1}}{c_{3}^{2}} c_{3}=c_{2} c_{3}>8 c_{2}$. Therefore, $c_{4}>0$. On the other hand, from $c_{3}>\frac{\kappa_{1}+\sqrt{\kappa_{1}^{2}+4 c_{1}^{3} \kappa_{1}}}{2 c_{1}^{2}}$, we have $c_{1}^{2} c_{3}^{2}-c_{3} \kappa_{1}-c_{1} \kappa_{1}>0$. Then, $c_{3}<\frac{c_{1}\left(c_{1} c_{3}^{2}-\kappa_{1}\right)}{\kappa_{1}}=c_{1}\left(\frac{c_{1}}{c_{2}}-1\right)$. Therefore, $c_{3}<\frac{c_{1}\left(c_{1}-c_{2}\right)}{c_{2}}=c_{4}$. Thus, $\frac{1}{c_{3}}-\frac{1}{c_{4}}>0$. Moreover, it is easy to check that the conditions (29) imply that the conditions (20) are satisfied. The proof is completed.

\section{Remark 4}

In this paper, $T_{\max }, \tau$ and $T_{\min }$ depend on the high gain $L\left(c_{2}, c_{3}, c_{4}, \rho_{2}\right.$ are constants which are independent on $L$ ). If the high gain $L$ is large, $T_{\max }$ and $\tau$ will be small to ensure the convergence. In fact, if the nonlinear terms $f_{i}\left(x_{1}, \cdots, x_{i}\right)$ change dramaticly, which means that $l_{1}$ is very large, then, $L$ will be given larger to dominate the nonlinear terms. Since $e_{1}\left(t_{k}\right)=x_{1}\left(t_{k}\right)-\hat{x}_{1}\left(t_{k}\right)$ is a constant on the interval $\left[t_{k}+\tau_{k}, t_{k+1}+\tau_{k+1}\right)$, then, $T_{k}$ and $\tau$ will be very small to ensure exponential convergence.

\section{Remark 5}

In [11], the authors described a class of continuous nonlinear systems at the sampled points by a discrete-time equation. Then, they considered multirate sampled-data output feedback control of the nonlinear systems without considering transmission delay. It should be noted that in some cases, it will be difficult to obtain an exact discrete time model for a nonlinear system. Not to mention that it is almost impossible to get an accurate approximation when there exist unknown nonlinear functions. In [17, 19], a state feedback law has been constructed to achieve global asymptotic stabilization for the nonlinear system (10) under sampled and delayed measurements, and with inputs subject to delay and zero-order hold. The nonlinear terms $f_{i}(\cdot)$ considered in $[17,19]$ are Lipschitz. The sampled-data feedback is based on a predictor mapping, which can be constructed inductively. In [13], a sampled-data output feedback stabilizer was presented to ensure that the closed-loop system is globally stable based on a hybrid system method. However, the closed-loop system is required to transformed into the hybrid system introduced in [20]. Whereas, the systems considered in this paper are in lower-triangular form with sampled and delayed measurements, and the nonlinear functions $f_{i}\left(x_{1}, \cdots, x_{i}\right)$ are unknown. The proposed output feedback stabilizer is continuous and hybrid. It has a simple and explicit form and can be derived without discretization.

Based on Theorem 2 and Corollary 3 , an algorithm sketch to set the design parameters is presented as follows. 
Step 1: We set the values of $a_{i}$ and $k_{i}$ such that (17) and (18) hold;

Step 2: We select $L$ such that the condition (19) is satisfied;

Step 3: Calculate $c_{1}$, select $\rho_{2}>2$ and $c_{3}$ such that $c_{3}>\max \left\{8,16 n \bar{a}_{1}\left(\lambda_{1}^{2}\left(\beta_{2}+1\right)+\lambda_{3}^{2}\right)+\right.$ $\left.c_{1}, \frac{\kappa_{1}}{c_{1}}, \frac{\kappa_{1}+\sqrt{\kappa_{1}^{2}+4 c_{1}^{3} \kappa_{1}}}{2 c_{1}^{2}}\right\}$, then calculate $c_{2}$ and $c_{4}$.

\section{Numerical simulation}

In this section, we use an example to show the effectiveness of our output feedback stabilization for nonlinear systems with sampled and time delay measurements.

\section{Example 1}

A single-link robot arm system can be modeled by [26] or [27]

$$
\left\{\begin{array}{l}
\dot{z}_{1}(t)=z_{2}(t), \\
\dot{z}_{2}(t)=\frac{K}{J_{2} N} z_{3}(t)-\frac{F_{2}(t)}{J_{2}} z_{2}(t)-\frac{K}{J_{2}} z_{1}(t)-\frac{m g d}{J_{2}} \cos \left(z_{1}(t)\right), \\
\dot{z}_{3}(t)=z_{4}(t) \\
\dot{z}_{4}(t)=\frac{1}{J_{1}} u(t)+\frac{K}{J_{1} N} z_{1}(t)-\frac{K}{J_{2} N} z_{3}(t)-\frac{F_{1}(t)}{J_{1}} z_{4}(t), \\
y=z_{1}(t),
\end{array}\right.
$$

where $J_{1}, J_{2}, K, N, m, g, d$ are known parameters, $F_{1}(t)$ and $F_{2}(t)$ are viscous friction coefficients that are not precisely known. Suppose $F_{1}(t)$ and $F_{2}(t)$ are bounded by an unknown constant $C>0$. We introduce the change of coordinates $x_{1}=z_{1}, x_{2}=z_{2}, x_{3}=\frac{K}{J_{2} N} z_{3}-\frac{m g d}{J_{2}}, x_{4}=\frac{K}{J_{2} N} z_{4}$ and the pre-feedback $v=\frac{K}{J_{2} N}\left(\frac{1}{J_{1}} u-\frac{m g d}{J_{2}}\right)$, which transforms (30) into

$$
\left\{\begin{array}{l}
\dot{x}_{1}(t)=x_{2}(t) \\
\dot{x}_{2}(t)=x_{3}(t)-\frac{F_{2}(t)}{J_{2}} x_{2}(t)-\frac{K}{J_{2}} x_{1}(t)-\frac{m g d}{J_{2}}\left(\cos \left(x_{1}(t)\right)-1\right) \\
\dot{x}_{3}(t)=x_{4}(t) \\
\dot{x}_{4}(t)=v+\frac{K^{2}}{J_{1} J_{2} N^{2}} x_{1}(t)-\frac{K}{J_{2} N} x_{3}(t)-\frac{F_{1}(t)}{J_{1}} x_{4}(t) \\
y(t)=x_{1}(t)
\end{array}\right.
$$

Construct the following output feedback controller

$$
\left\{\begin{array}{l}
\dot{\hat{x}}_{1}(t)=\hat{x}_{2}(t)+a_{1} L\left(y\left(t_{k}\right)-\hat{x}_{1}\left(t_{k}\right)\right), \\
\dot{\hat{x}}_{2}(t)=\hat{x}_{3}(t)-\frac{F_{2}(t)}{J_{2}} \hat{x}_{2}(t)-\frac{K}{J_{2}} \hat{x}_{1}(t)-\frac{m g d}{J_{2}}\left(\cos \left(\hat{x}_{1}(t)\right)-1\right)+a_{2} L^{2}\left(y\left(t_{k}\right)-\hat{x}_{1}\left(t_{k}\right)\right), \\
\dot{\hat{x}}_{3}(t)=\hat{x}_{4}(t)+a_{3} L^{3}\left(y\left(t_{k}\right)-\hat{x}_{1}\left(t_{k}\right)\right), \\
\dot{\hat{x}}_{4}(t)=v+\frac{K^{2}}{J_{1} J^{2} N^{2}} \hat{x}_{1}(t)-\frac{K}{J_{2} N} \hat{x}_{3}(t)-\frac{F_{1}(t)}{J_{1}} \hat{x}_{4}(t)+a_{4} L^{4}\left(y\left(t_{k}\right)-\hat{x}_{1}\left(t_{k}\right)\right), \\
u(t)=-\left[k_{1} L^{4} \hat{x}_{1}(t)+k_{2} L^{3} \hat{x}_{2}(t)+k_{3} L^{2} \hat{x}_{3}(t)+k_{4} L \hat{x}_{4}(t)\right], \quad t \in\left[t_{k}+\tau_{k}, t_{k+1}+\tau_{k+1}\right), \\
\hat{x}_{i}\left(t_{k+1}+\tau_{k+1}\right)=\lim _{t \rightarrow t_{k+1}+\tau_{k+1}-\hat{x}(t), \quad i=1, \cdots, 4, k \geq 0 .}
\end{array}\right.
$$

In the following simulation, we apply the system parameters: $K / J_{2}=5, \mathrm{mgd} / J_{2}=4$, $K^{2} /\left(J_{1} J_{2} N^{2}\right)=2, K /\left(J_{2} N\right)=3, F_{1}(t) / J_{1}=10, F_{2}(t) / J_{2}=10$ and $L=2$. The control gain $k_{1}=40, k_{2}=78, k_{3}=49, k_{4}=12$ and the observer gain $a_{1}=4, a_{2}=6, a_{3}=4, a_{4}=1$. The initial conditions of the whole system are $\left(x_{1}(0), x_{2}(0), x_{3}(0), x_{4}(0),\right)=(-5,-1,4,20)$ and $\left(\hat{x}_{1}(0), \hat{x}_{2}(0), \hat{x}_{3}(0), \hat{x}_{4}(0),\right)=(5,3,-1,-4)$. The sampling period $T_{k}$ and the delay $\tau_{k}$ are given as $T_{k}=0.1 \mathrm{~s}$ and $\tau_{k}=0.05 \mathrm{~s}$, respectively. The simulation results are shown in Fig. 1 . 


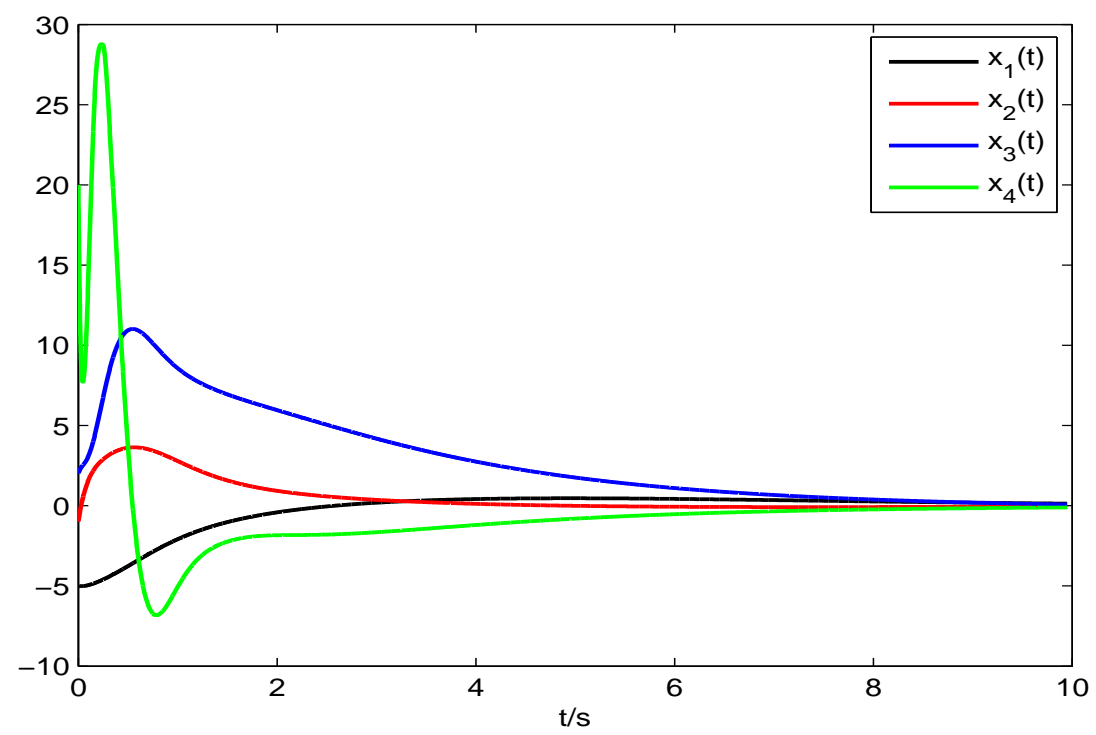

Fig. 1. Trajectories of the states $x_{i}(t)(1 \leq i \leq 4)$ with the output feedback stabilizer.

\section{Conclusion}

In this paper, we addressed the problem of output feedback stabilization for nonlinear systems with sampled and delayed output measurements. Firstly, sufficient conditions were proposed to ensure that a class of hybrid systems are globally exponentially stable. Then, based on the sufficient conditions and a dedicated construction continuous observer, an output feedback control law was presented to globally exponentially stabilize the nonlinear systems. The output feedback stabilizer was continuous and hybrid, and could be derived without discretization. The maximum allowable sampling period and the maximum delay were also given.

\section{Acknowledgments}

This work was supported by the National Science Foundation of China $(61174216,61374028$, 61374171), the Grant National Science Foundation of Hubei Provincial (2013CFA050), the Scientific Innovation Team Project of Hubei Provincial Department of Education (T201504).

\section{References}

1. Praly L, Asymptotic stabilization via output feedback for lower triangular systems with output dependent incremental rate. IEEE Trans. Automat. Contr. 2003; 48(6): 1103-1108.

2. Esfandiari F and Khalil H K, Output feedback stabilization of fully linearizable systems. Int. J. Control 1992; 56(5): 1007-1037.

3. Lin $\mathrm{Z}$ and Saberi A, Robust semi-global stabilization of minimum-phase input-output linearizable systems via partial state and output feedback. IEEE Trans. Automat. Contr. 1995; 40(6): 1029-1041.

4. Teel A R and Praly L, Tools for semiglobal stabilization by partial state and output feedback. SIAM Journal Contr. and Optimiz. 1995; 33(5): 1443-1448.

5. Praly L and Jiang Z, Linear output feedback with dynamic high gain for nonlinear systems. Syst. Control Lett. 2004; 53(2): 107-116.

6. Arcak M and Nešić D, A framework for nonlinear sampled-data observer design via approximate discrete-time models and emulation. Automatica 2004; 40(11): 1931-1938.

7. Barbot J P, Monaco S and Normand-Cyrot D, Discrete-time approximated linearization of SISO systems under output feedback. IEEE Trans. Automat. Contr. 1999; 44(9): 1729-1733.

8. Khalil H K, Performance recovery under output feedback sampled-data stabilization of a class of nonlinear systems. IEEE Trans. Automat. Contr. 2004; 49(12): 2173-2184. 
9. Nešić D and Teel A R, A framework for stabilization of nonlinear sampled-data systems based on their approximate discrete-time model. IEEE Trans. Automat. Contr. 2004; 49(7): 1103-1122.

10. Nešić D, Teel A R and Kokotović P V, Sufficient conditions for stabilization of sampled-data nonlinear systems via discrete-time approximations. Syst. Control Lett. 1999; 38(4): 259-270.

11. Ahrens J H, Tan X and Khalil H K, Multirate sampled-data output feedback control with application to smart material actuated systems.IEEE Trans. Automat. Contr. 2009; 54(11): 2518-2529.

12. Clarke F H, Ledyaev Y S, Sontag E D and Subbotin A I, Asymptotic controllability implies feedback stabilization. IEEE Trans. Automat. Contr. 1997; 54(11): 1394-1407.

13. Nešić D, Teel A R and Carnevale D, Explicit computation of the sampling period in emulation of controllers for nonlinear sampled-data systems. IEEE Trans. Automat. Contr. 2009; 54(3): 619-624.

14. Nadri H, Hammouri $\mathrm{H}$ and Mota R, Observer design for uniformly observable systems with sampled measurements. IEEE Trans. Automat. Contr. 2013; 58(3): 757-762.

15. Ahmed-Ali T, Karafyllis I and Lamnabhi-Lagarrigue F, Global exponential sampled-data observers for nonlinear systems with delayed measurements. Syst. Control Lett. 2013; 62(7): 539-549.

16. Zhang D, Shen Y and Xia X, Continuous observer design for nonlinear systems with sampled and delayed output measurements. in 19th IFAC World Congress. Cape Town, South Africa, 2014: 269-274.

17. Karafyllis I and Krstic M, Nonlinear stabilization under sampled and delayed measurements, and with inputs subject to delay and zero-order hold. IEEE Trans. Automat. Contr. 2012; 57(5): 1141-1154.

18. Heemels H, Teel A R, Wouw N and Nešić D, Networked control systems with communication constraints: tradeoffs between transmission intervals, delays and performance. IEEE Trans. Automat. Contr. 2010; 55(8): 1781-1796.

19. Ahmed-Ali T and Karafyllis I, Krstic M and Lamnabhi-Lagarrigue F, Robust stabilization of nonlinear globally Lipschitz delay systems, Recent results on nonlinear time delayed ssytems (Eds. Malisoff M, Mazenc F, Pege P and Karafyllis I) in the series advances in delays and dynamics (ADD@ S), Spinger, 2015.

20. Goebel R and Teel A R, Solution to hybrid inclusions via set and graphical convergence with stability theory applications. Automatica 2006: 42(4): 573-587.

21. Karafyllis I, A system-theoretic framework for a wide class of systems I: Applications to numerical analysis. $J$. Math. Anal. 2007; 328(2): 876-899.

22. Karafyllis I, A system-theoretic framework for a wide class of systems II: Input-to-output stability. J. Math. Anal. 2007; 328(1): 466-486.

23. Karafyllis I and Jiang Z, P, A small-gain theorem for a wide class of feedback systems with control applications. SIAM Journal Contr. and Optimiz. 2007; 46(4): 1483-1517.

24. Ahmed-Ali T, Van Assche V, Massieu J and Dorleans P, Continuous-discrete observer for state affine systems with sampled and delayed measurements. IEEE Trans. Automat. Contr. 2013; 58(4): 1085-1091.

25. Liu Y, Wang Z and Liu X, On global exponential stability of generalized stochastic netural networks with mixed time-delays. Neurocomputing 2006; 70(1): 314-326.

26. Isidori, Nonlinear Control Systems, New York: Springer, 1995.

27. Marino R and Tomei P, Nonlinear Control Design, Uk: Prentice Hall International, 1995.

28. Tsinias J, A theorem on global stabilization of nonlinear systems by linear feedback. Syst. Control Lett. 1991; 17: 357-362.

29. Gauthier J, Hammouri $\mathrm{H}$ and Othman S, A simple observer for nonlinear systems applications to bioreactors. IEEE Trans. Automat. Contr. 1992; 37: 875-880.

30. Lei $\mathrm{H}$ and Lin W, Universal adaptive control of nonlinear systems with unknown growth rate by output feedback, Automatica, 2006; 42: 1783-1789.

31. Coddington E A and Levinson N, Theory of Ordinary Differential Equations, McGraw-Hill, New York, 1955. 